\title{
An analysis of experimental techniques for estimation of dissipated energy evolution in metal alloys under plastic deformation
}

\author{
by O. Plekhov, A. Vedernikova, A. Iziumova, A. Vshivkov
}

Institute of Continuous Media Mechanics of the UB RAS, Academika Koroleva st, 1, 614013, Perm, Russian Federation, poa@icmm.ru

\begin{abstract}
This work is devoted to the comparative analysis two approaches for investigation of energy dissipation in metal under fatigue crack propagation. The flat specimens with V-shaped stress concentrators from steel 304 AISE and titanium alloy Grade 2 were tested. The dissipated energy was measured using both original contact heat flux sensor and IR camera FLIR SC5000. The results of IR monitoring were analysed with a goal to estimate the amplitude of second temperature harmonics (D-mode). As a result of the study, we have shown that the dissipated energy values estimated by both methods have a good qualitative agreement. The measured values allows us to propose a correlation between the crack rate and dissipated energy for predicting the fatigue crack growth.
\end{abstract}

\section{Introduction}

One of the actual problems of the fracture mechanics is the forecast of lifetime of cracked metal parts of engineering constructions subjected to the cyclic loading. The process of fatigue crack propagation in metals is accompanied by intense strain localization in areas of monotonic and cyclic plastic deformation leads to the temperature perturbation at crack tip. Many authors investigated the correlation between crack propagation rate and intensity of energy storage and dissipation processes [1-20] and showed that the dissipated energy plays a key role in the crack growth. From experimental point of view, the dissipated energy is well-measured value and could be estimated by different experimental techniques.

One of them is based on the application of original contact measurement system based on Peltier elements that allows providing quantitative integral values of heat flow in some area near crack tip. Such methodology was originally used for studying the energy dissipation under liquid flow [3] as well as the failure of metals [2, 4].

The second way for calculated of the power of the heat flow based on the analysis of evolution of the temperature field on the specimen surface by means IR thermography data. Heat sources field calculation is carried out with the using of heat conduction equation averaged on volume. This calculation is usually associated with the need to differentiate strongly oscillating signals and to determine the parameters responsible for the interaction of the specimen with environment. The IR data widely used to gather information for a better understanding of plastic deformation and fracture of metallic materials [5-10].

In works $[11,12]$ shows the satisfactory agreement of the results contact (heat flux sensor) and non-contact (IR thermography) measurements of dissipated energy during irreversible deformation. Proved the possibility using of these measurements in a combination (to verify the heat source value, its distribution over the material surface and heat exchange conditions for specimen and environment) or separately (as an express method to evaluate material conditions at different stages of loading).

The third way is Lock-in thermography that allows space-resolved measurements and directly evaluate of elastic stress fields according to the thermo-elastic effect [13] and the investigation of dissipative energies with the double frequency method proposed by Sakagami [14]. The determination of dissipated energy is used to evaluate the crack initiation as well as effects in crack propagation experiments [15-20].

In this study, we have shown that the dissipated energy values estimated by Lock-In thermography technique and contact heat flux sensor have a good qualitative agreement.

Both techniques could be applied for quasi-static and cyclic loading. The study of quasi-static loading allows us to show that the stored energy rate peaks on the initial stage of plastic deformation and vanishes at developed plastic flow while the value of the stored energy reaches a certain critical value that means that materials prepared to the failure. The focus of this work is cyclic loading. The measured values dissipated energy under cyclic loading allows us to determine a linear correlation between the crack rate and dissipated energy (value of amplitude of second temperature harmonics) for predicting the fatigue crack growth.

\section{Experimental details}

A series of V-notched flat specimens made from stainless steel AISE 304 and titanium alloy Grade 2 were tested in a servohydraulic testing machine Instron 8802 and electro-mechanical testing machine Shimadzu AG-X Plus. During crack propagation experiments the specimens were subjected to cyclic loading at a frequency $10 \mathrm{~Hz}$ with constant stress amplitude and $\mathrm{R}=0$. For crack length measurement the DC potential drop method was used [21]. 
To analyse the heat flow power at the crack tip the Seebeck effect-based heat flux sensor developed in previous study was used [2]. To enhance the heat flow, a heat-conductive paste was applied between the sensor and the specimen.

The evolution of the temperature field was recorded in some time intervals by an IR camera FLIR SC 5000. IR camera has the following features: the spectral range of 3-5 $\mu \mathrm{m}$, the maximum frame size is $320 \times 256$ pixels, the spatial resolution is $10^{-4}$ meters. The temperature sensitivity is in the range from $25 \mathrm{mK}$ to $300 \mathrm{~K}$. Calibration of the camera was made based on the standard calibration table. The surface of the specimens intended for infrared shooting was polished in several stages and coated by a thin layer of amorphous carbon to enhance the emissivity.

The thermography sequences with a length of $10 \mathrm{~s}$ at a frame rate $100 \mathrm{~Hz}$ were recorded and after analysed with the infrared imaging software suite Altair LI.

The geometry of specimens and experimental setup are shown in Fig. 1-2, dimension are reported in $\mathrm{mm}$. In table 1 are presented chemical composition for both materials.

Table 1. Chemical composition of materials.

\begin{tabular}{|c|c|c|c|c|c|c|c|}
\hline \multicolumn{7}{|c|}{ Grade 2, \% } \\
\hline $\mathrm{Fe}$ & $\mathrm{C}$ & $\mathrm{Si}$ & $\mathrm{N}$ & $\mathrm{Ti}$ & $\mathrm{O}$ & $\mathrm{H}$ & Other \\
\hline 0.25 & 0.07 & 0.1 & 0.04 & $99.24-99.7$ & 0.2 & 0.01 & 0.3 \\
\hline \multicolumn{7}{|c|}{ AISE 304, \% } \\
\hline $\mathrm{C}$ & $\mathrm{Cr}$ & $\mathrm{Fe}$ & $\mathrm{Mn}$ & $\mathrm{Ni}$ & $\mathrm{P}$ & $\mathrm{S}$ & $\mathrm{Si}$ \\
\hline 0.08 & $18-20$ & $66.34-74$ & 2 & $8-10.5$ & 0.045 & 0.03 & 1 \\
\hline
\end{tabular}

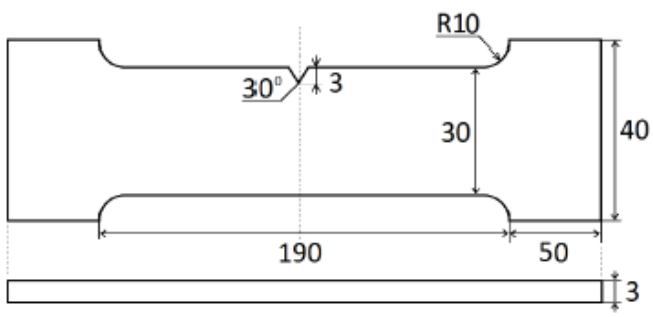

Fig. 1: Geometry of specimens.

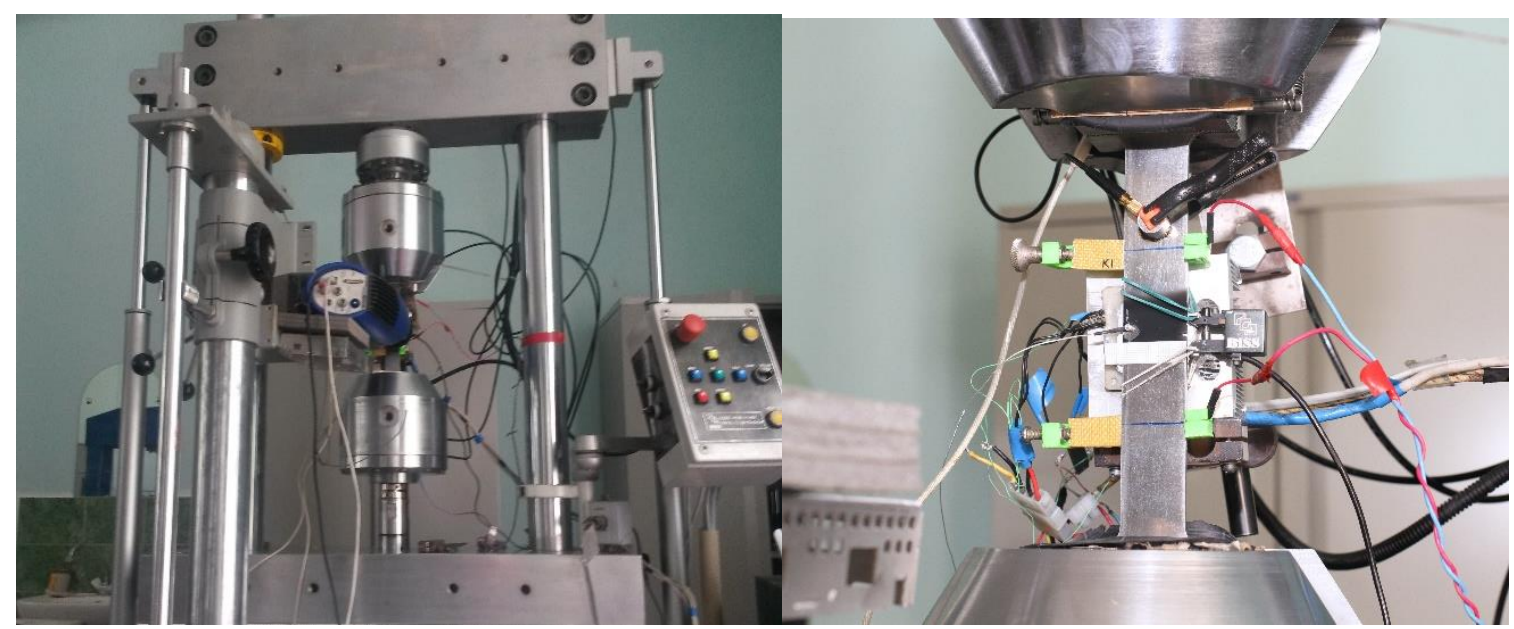

Fig. 2: Experimental setup used for testing.

\section{Heat flux sensor measurements}

To analyse the energy balance at the crack tip a contact heat flux sensor was used [2]. The proposed sensor is based on the Seebeck effect, which is the reverse of the Peltier effect. The contact heat flux sensor is attached to the specimen during all mechanical test. To enhance the heat flow a heat-conductive paste was inserted between the sensor and the specimen. The quantity of heat absorbed or dissipated by the element is directly proportional to the current intensity and the time of its passage: 


$$
P=\Pi_{A B} I
$$

where $P$ - the power of heat flow, $I$ - the direct current, $\Pi_{A B}$ - Peltier coefficient, which is related with a coefficient of thermal electromotive force.

The signal from the flux sensor is measured by the amplifier and registered in the ADC of the microcontroller. The data are transmitted from the microcontroller to the personal computer for further processing. Sensors were calibrated using a device with a controlled heat flow. The heat insulating system provides the heat flow from the resistance to the sensor only. The heat flow was calculated using the values of the resistor voltage and the electric current across the resistor.

\section{Lock-in thermography measurements}

The dissipated energy can be estimated by Lock-in thermography. Lock-in thermography is based on a correlation in frequency, amplitude and phase of the detected signal with a reference signal coming from the loading system. The evaluation is based on a Discrete Fourier Transformation (Eqn. 2) and performed for each pixel of the recorded frames. It was shown that in case of plastic deformation a second mode coupled with the double loading frequency appears (Dmode) and correlated with dissipative energies [14]. For each analysed sequence of frames, the evaluation provides an amplitude and a phase image for the different modes.

$$
T(t)=T_{m}+T_{E} \cdot \sin \left(2 \pi f_{L} \cdot t+\varphi_{E}\right)+T_{D} \cdot \sin \left(2 \cdot 2 \pi f_{L} \cdot t+\varphi_{D}\right)
$$

where $T_{m}$ - mean temperature, $f_{L}$ - mechanical loading frequency, $\varphi_{E}$ and $\varphi_{D}$ - phase shifts, $T_{E}$ - thermo-elastic amplitude (E-mode), $T_{D}$ - plasticity effects amplitude (D-mode).

Eq. (1) is integrated in the algorithm of Altair LI software, providing an image in data matrix form for each constant parameter. In figure 3 the resulting D-amplitude images of the lock-in evaluation of an experiment with a constant stress amplitude and a crack length of about $7 \mathrm{~mm}$ for both materials are shown. The D-amplitude image shows the plastic zone in front of the crack tip.

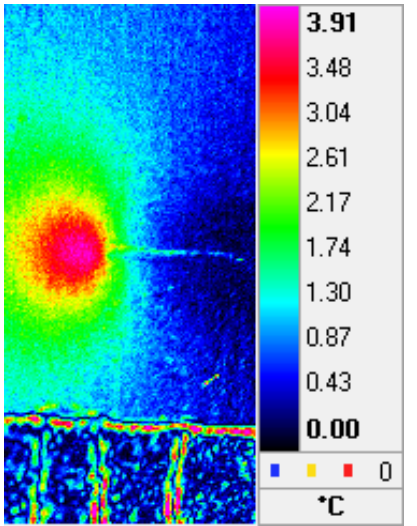

(a)

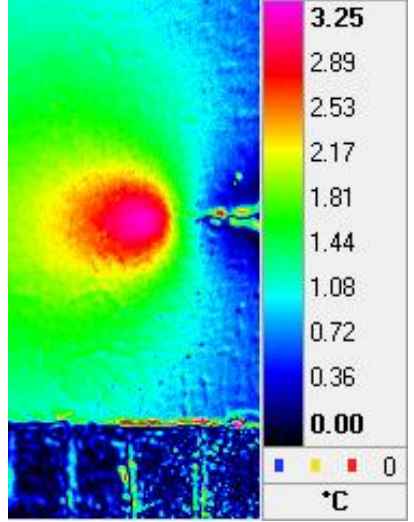

(b)

Fig. 3: D-Amplitude image of a specimens loaded with a constant stress amplitude for (a) Grade 2, (b) steel AISE 304.

\section{Results}

To compare the heat flux sensor results and the results of Lock-in thermography measurements were have studied the temperature evolution in small area covered all temperature fluctuation near crack tip. The mean values of the amplitude coupled to the double loading frequency (D-mode) was determined using t Altair LI software. Figure 4 shows the results of the normalized thermographic and the heat flux sensor measurements of a crack propagation experiment with a constant force for stainless steel AISE 304 and titanium alloy Grade 2 specimens. Result of measurements of the heat flux during the experiment by contact sensor can be divided into three parts. Short initial increasing part corresponds to starting of crack propagation. The second part with constant heat flux corresponds to the regime of short crack propagation. The last part of the plot is characterized by sharp increasing of heat dissipation. During this part we observe the long crack propagation process. It can be see that the dissipated energies measured by both methods have a good qualitative agreement for period with high heat dissipation. For both investigated materials, the averaged D-Amplitude values are rising with loading cycles what can be caused by processes in front of the crack tip. 
The D-mode amplitude is correlated to the energy of irreversible process and increases toward loading cycles. The same behaviour have the crack rate toward loading cycles. Therefore, the value of $D$-mode amplitude measurements can be used for describing the crack propagation. In figures 5, 6 relation between D-mode variation and crack growth rate for both materials are presented. A linear relation can be determined between parameters and can be used for predicting the fatigue crack growth:

$$
d a / d N=a S_{d}^{b}
$$

where $S_{d}$ - the maximum amplitude of the thermal signal that changes at the double of loading frequency.

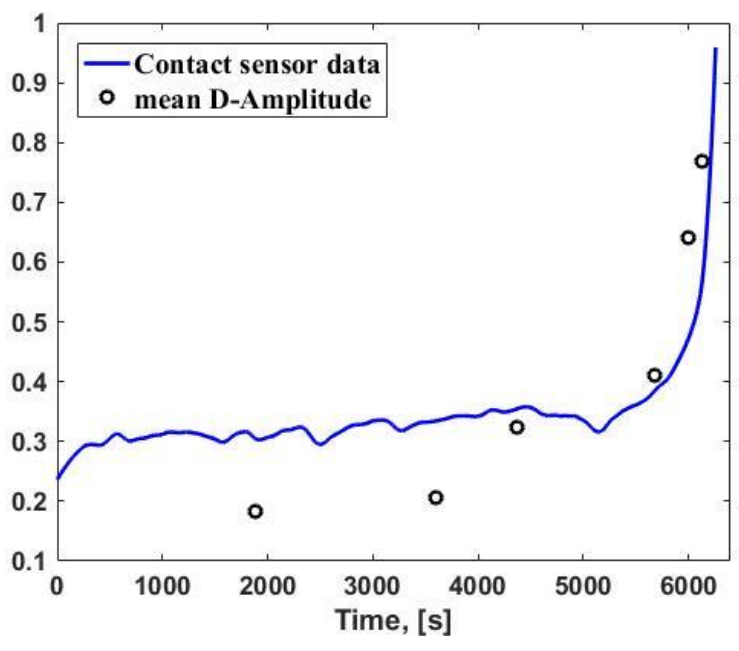

(a)

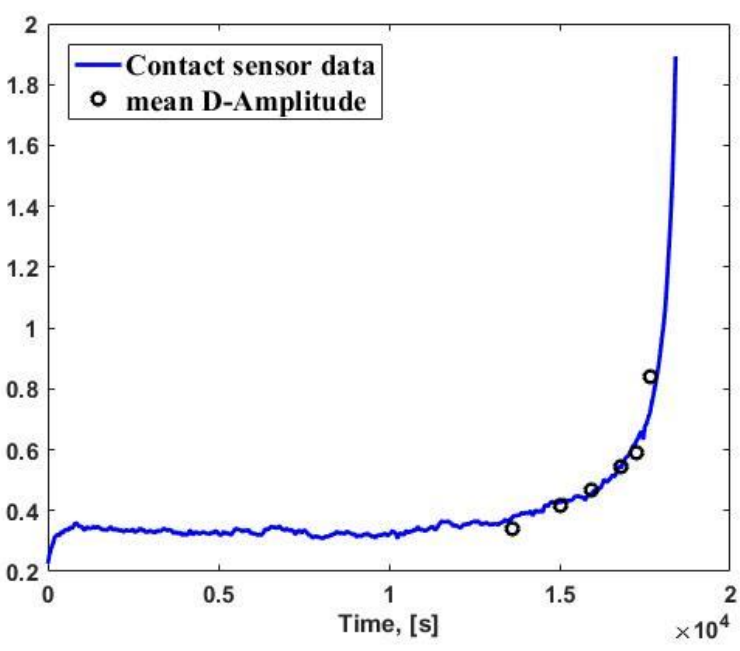

(b)

Fig. 4: Results of thermographic and heat flux sensor measurements for (a) Grade 2 specimen of an experiment with a constant force of $F=5 \mathrm{kN}$, (b) steel AISE 304 specimen of an experiment with a constant force of $F=6 \mathrm{kN}$.

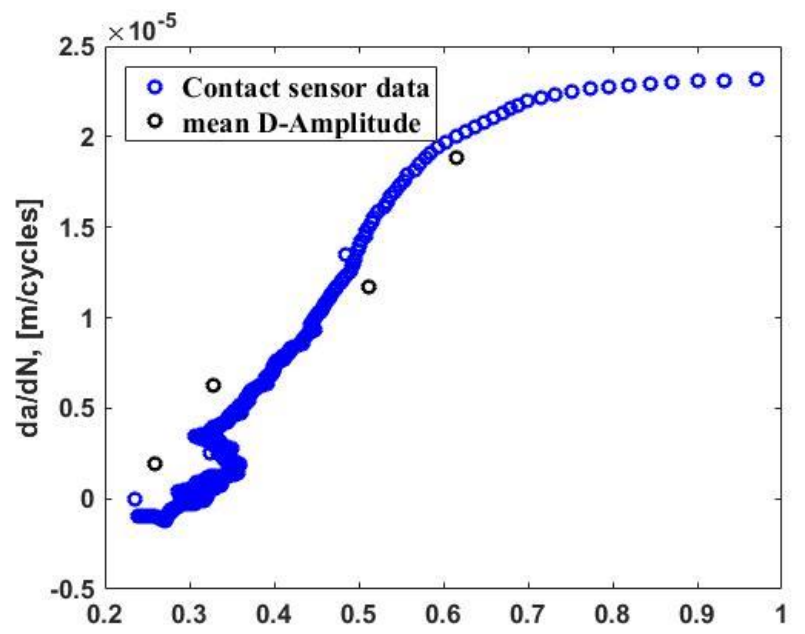

(a)

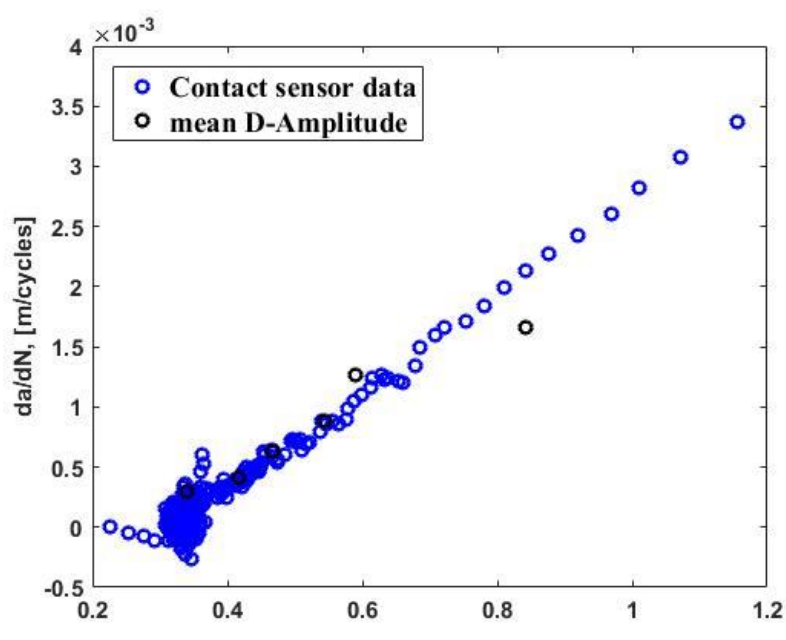

(b)

Fig. 5: Relation between amplitude double load frequency and crack growth rate for (a) Grade 2 specimen of an experiment with a constant force of $F=5 \mathrm{kN}$, (b) steel AISE 304 specimen of an experiment with an constant force of $F=6 \mathrm{kN}$ 


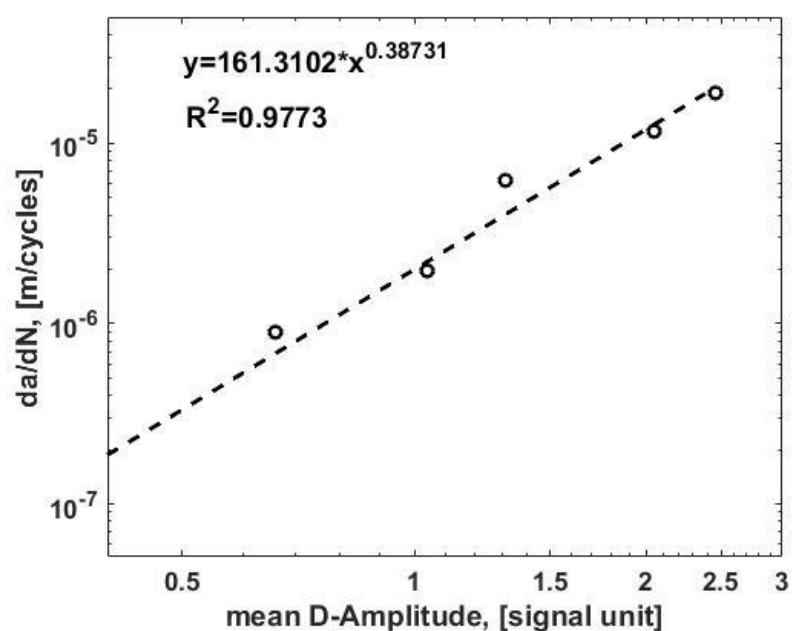

(a)

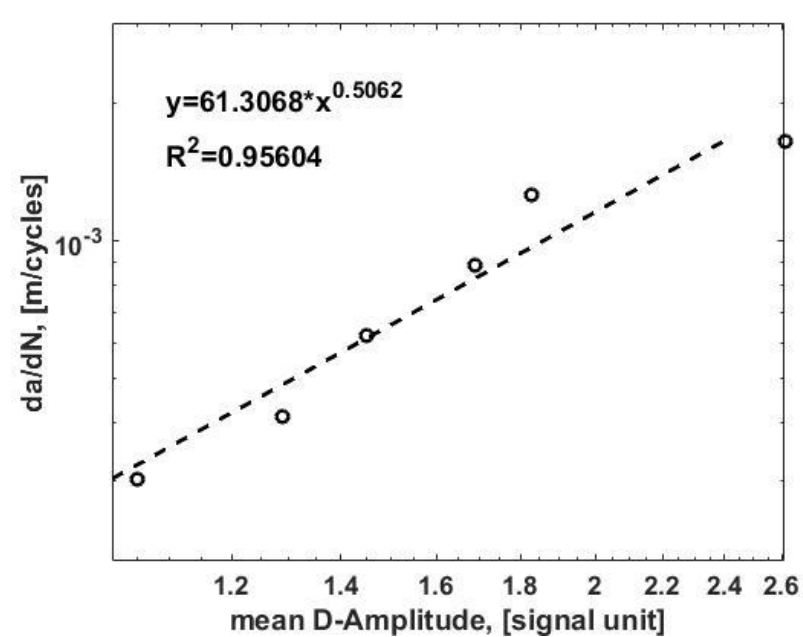

(b)

Fig. 6: Relation between second order temperature variation and crack growth rate for (a) Grade 2 specimen of an experiment with a constant force of $F=5 \mathrm{kN}$, (b) steel AISE 304 specimen of an experiment with an constant force of $F=6 \mathrm{kN}$.

\section{Conclusion}

The thermographic and heat flux sensor measurements for estimation of dissipated energy and predicting the fatigue crack growth were used. A good qualitative agreement between values dissipated energies measured by both methods was obtained. With increasing the crack length the thermographic and flux sensor measurements showed an increase of the dissipated energies measured in front of the crack tip. The measured values dissipated energy allows us to determine a linear correlation between the crack rate and dissipated energy (value of amplitude of second temperature harmonics).

\section{Acknowledgments}

This work was supported by the grant of the Russian Foundation for Basic research №18-31-00293, 16-51-48003.

\section{REFERENCES}

[1] Iziumova A, Plekhov O. Calculation of the energy J-integral in plastic zone ahead of a crack tip by infrared scanning. FFEMS. 2014;37:1330-1337.

[2] Vshivkov A, Iziumova A, Bar U, Plekhov O. Experimental study of heat dissipation at the crack tip during fatigue crack propagation. Fract Struct Integr. 2016;35:131-137.

[3] Pradere C, Joanicot M, Batsale J-C, Toutain J, Gourdon C. Processing of temperature field in chemical microreactors with infrared thermograph. QIRT Journal. 2006;3:117-135.

[4] Wang C, Blanche A, Wagner D, Chrysochoos A, Bathias C. Dissipative and microstructural effects associated with fatigue crack initiation on an Armco iron. Int J Fatigue. 2014;58:152-7.

[5] Iziumova A, Plekhov O, Vshivkov A, Prokhorov A, Uvarov S. Studying the Rate of Heat Dissipation at the Vertex of a Fatigue Crack. Tech Phys Lett. 2014;40:810-812.

[6] lino Y. Fatigue crack propagation work coefficient - a material constant giving degree of resistance to fatigue crack growth. Eng Fract Mech. 1979;12:279-299.

[7] Chow C, Lu T. Cyclic J-integral in relation to fatigue crack initiation and propagation. Eng. Fract. Mech. 1991;39:120.

[8] Dowling N, Begley J. Fatigue Crack Growth During Gross Plasticity and the J-Integral. Mechanics of Crack Growth, ASTM STP 590, American Society for Testing and Materials. 1976:82-103.

[9] Fedorova A, Bannikov M, Terekhina A, Plekhov O. Heat dissipation energy under fatigue based on infrared data processing. QIRT J. 2014;11(1):2-9.

[10] Plekhov O, Saintier N, Palin-Luc T, Uvarov SV, Naimark OB. Theoretical analysis, infrared and structural investigations of energy dissipation in metals under cyclic loading; 2007. Mat Sci Eng. 2007;462(1-2):367-369.

[11] Iziumova A, Vshivkov A, Prokhorov A, Plekhov O, Venkatraman B. Study of heat source evolution during elasticplastic deformation of titanium alloy Ti-0.8Al-0.8Mn based on contact and non-contact measurements. PNRPU Mechanics Bulletin. 2016;1:68-81. 
[12] Plekhov O, Vshivkov A, Iziumova A, Zakharov A, Shlyannikov V. The experimental study of energy dissipation during fatigue crack propagation under biaxial loading. Frattura ed Integrità Strutturale. 2019;48:50-57.

[13] Brémond P. New developments in Thermo Elastic Stress Analysis by Infrared Thermography. IV Pan-American Conference for Non Destructive Testing, Buenos Aires, 2007.

[14] Sakagami T, Kubo S, Tamura E, Nishimura T. Identification of plastic-zone based on double frequency lock-in thermographic temperature measurement. In: International conference of fracture ICF 11, 2005.

[15] Bar J. Determination of dissipated energy in fatigue crack propogetion experiments with Lock-in thermography and heat flow measurements. Proc Structl Int. 2016;2:2105-2112.

[16] De Finis R, Palumbo D, Ancona F, Galietti U. Fatigue behaviour of stainless steels: a multi-parametric approach. Proc. of the Society for Experimental Mechanics Series. 2016;9:1-8.

[17] De Finis R, Palumbo D, Galietti U. Energetic approach to study the plastic behaviour in CT specimens. In: Proceedings of QIRT; 2018; Berlin, Germany. Available from QIRT Open Archives: http://qirt.gel.ulaval.ca/dynamique/index.php?idD=81\&Lang=0. Paper QIRT 2018-133.

[18] Bär J, Seifert S. Investigation of energy dissipation and plastic zone size during fatigue crack propagation in a high-alloyed steel. Procedia Mater Sci 2014;3:408-13.

[19] Bär J, Seifert S. Thermographic investigation of fatigue crack propagation in a high-alloyed steel. Adv Mater Res 2014;891-892:936-41.

[20] Bär J. Determination of dissipated energy in fatigue crack propagation experiments with lock-in thermography. Procedia Struct Integrity 2016;2:2105-12.

[21] Nayeb-Hashemi H, Swet D, Vaziri A. New electrical potential method for measuring crack growth in nonconductive materials. Measurement. 2004;36(2):21-129. 